\section{Validation of the Use of Trans- cutaneous Electrical Nerve Stimulation for Patients with Pain and Recommendations for its Use by Physiotherapists}

\section{Phyllis Berger*}

Department of Health Sciences, University of the Witwatersrand, Johannesburg, South Africa

\begin{abstract}
Although Transcutaneous Electrical Nerve Stimulation (TENS) has been in use since the 1960's, it has been difficult to ascertain its effectiveness within evidence based clinical trials. This often impacts upon patient's wishing to acquire a device with support from their medical health schemes attributable to the lack of strong evidence. This is due to differences in the frequencies, pulse widths and modes of the various devices, the duration of application, areas of application, types of pain to which TENS is applied and even differences in patient responses to TENS. All of the above obscures assessment of its value however it has been observed that many patients in fact do respond to TENS. An attempt to evaluate the effectiveness of TENS has been made with recommendations for its use to both physiotherapists and other medical personnel that may apply this device for pain relief.
\end{abstract}

Keywords: Applications; Pain relief; Research; Tens

\section{Introduction}

Transcutaneous Electrical Nerve Stimulation known as TENS, refers to a small battery operated device that provides low voltage electrical impulses via surface electrodes to the skin, for the purpose of pain relief by modifying pain perception [1].

TENS has been used to relieve nociceptive and certain types of neuropathic pain since the 1960's [2]. It was the 'gate control' theory of that helped partly explain the mechanisms that may be involved when TENS is applied [3]. These mechanisms involve the A delta fibres (pain) and the A beta fibres (touch, pressure and vibration) in the sensory neurons that carry information from both the site of injury and

*Corresponding author: Phyllis Berger, Department of Health Sciences, University of the Witwatersrand, Johannesburg, South Africa, Tel: +27 824117777; E-mail: pberger@icon.co.za

Citation: Berger P (2017) Validation of the Use of Transcutaneous Electrical Nerve Stimulation for Patients with Pain and Recommendations for its Use by Physiotherapists. J Anesth Clin Care 4: 019.

Received: December 06, 2016; Accepted: February 12, 2017; Published: February 27, 2017 the two sites in the dorsal horn of the spinal cord. These are the inhibitory cells and the transmission cells. Signals from both the A delta and the A beta fibres excite the transmission cells and if the output of the transmission cells exceeds a critical level, pain begins. Inhibitory cells inhibit the activation of the transmission cells - known as the pain 'gate. The A delta fibres tend to impede the inhibitory cells leaving the gate open while the large diameter fibres excite the inhibitory cells tending to close the gate. It has been proposed that TENS therefore activates the A beta fibres to close the gate by inhibiting transmission of the A delta fibres and this has been proposed as one of the mechanisms involved in modification of pain transmission.

Since the1990s, TENS studies have demonstrated the ability to impact upon pain and this has became apparent, as patient satisfaction was high in surveys among chronic pain patients. However the results in randomized controlled trials were often non-significant and it appears that science has failed to demonstrate the effectiveness of TENS both in the treatment of pain and the differences between TENS and placebo.

This is not surprising as according to Bennett, Hughes \& Johnson [4], "the validity of many previous reviews on TENS are questionable". But their questions also have broader relevance for what methodology we use to assess physical modalities in pain treatment. "From their analysis of three Cochrane reviews and the 38 included trials, they found significant sources of bias in both directions. Traditional sources of bias, such as lack of inadequate randomization, blinding, and unaccounted withdrawals or dropouts that contribute to inflating effect estimates are familiar to most pain researchers".

"But their finding that low fidelity in TENS trials may confound results in a negative direction, (i.e., indicating lack of efficacy) is new and important". "This discrepancy can also be found in TENS trials investigating acute pain conditions, where non-significant VAS pain reduction scores of TENS versus placebo were reported, in spite of significantly higher patient satisfaction in the experimental, i.e., TENS, group" [5]. "Sometimes non-significant pain relief on VAS from TENS" can be readily explained by confounding factors, "such as $35 \%$ lower consumption of patient-administered analgesics after surgery" [6,7]. But Bennett, Hughes \& Johnson also points at "other sources of bias, such as the inappropriate timing of outcome measures, sub-optimal dosing and the fact that effective co-interventions are prevalent in many TENS trials" [4].

The material in Bennet et al., analysis was "collected from three inconclusive Cochrane reviews of TENS as previously mentioned. Protocols in these reviews did not address possible confounders of fidelity. Lack of fidelity assessments is unfortunately common in Cochrane reviews of physical modalities". "In fact, in a 1997 Cochrane review of TENS was, in spite of some limitations, considered effective for chronic low back pain. The review group was then changed and the subsequent 2001 update found no support for the use of TENS, based on five trials according to Koke et al. [8]. "Two trials from the 1997 version were excluded in the 2001 update. According to Lewis, that the Cochrane meta-analysis lacked data as to how TENS effectiveness is affected by type of application, site of application, treatment duration, optimal frequencies and intensities" [9]. "Still the next updated 
version in 2005 appeared with a negative conclusion and another change in exclusion criteria and only two trials were included" [10]. "The last and fourth (2009) version included two new trials and sustained the negative conclusion (now based on four trials). Only one TENS trial was included in all versions of the low back pain review [11], "Nevertheless, the same trial was excluded from another Cochrane review because TENS data were combined from two TENS groups receiving different (and potentially effective) co-interventions" [12]. These examples show how fidelity issues are handled differently leading to changes in Cochrane conclusions about TENS and continuance of the confusion.

"According to a systematic review to determine the effectiveness of TENS in the management of chronic low back pain by a consensus concluded that although it appears that there is limited and inconsistent evidence for the management of chronic low back pain as an isolated intervention, larger multi-centre randomised and controlled trials may resolve the true effectiveness of TENS [13]. In this same vein, the level of hypoalgesic efficacy of TENS appears to be dependent on TENS parameter combination selection (defined in terms of intensity, frequency and stimulation site) and the experimental pain model with future TENS trials employing these dose responses" [14].

"Regarding the timing of outcome measures, there is a general consensus that optimal benefit is achieved during TENS stimulation" [15]. "The onset of pain-reduction is rapid during TENS treatment, with effects reaching significance after 20-30 min, "optimal effects occuring after $40 \mathrm{~min}$ of TENS" $[16,17]$. "When TENS treatment is stopped, then the pain-relieving effect dissipates rapidly, within the first 30 min after a single treatment" [15]. "Repeating TENS treatment daily for 2 weeks may partly prolong the pain-relieving effect for a few weeks" [17]. "Despite this post-treatment effect, it is clear that optimal benefit from TENS is achieved during treatment, not after. Although several TENS trials have measured treatment effects beyond the period where TENS is most effective, the value of the results from these analyses is questionable. Just as it would be meaningless to evaluate the effects of Non-Steroidal Anti-Inflammatory Drugs (NSAID) in knee osteoarthritis a few days after the end of NSAID-treatment, when only non-significant effects of NSAIDS are reported [18], so perhaps it may not be suitable to study the effects of TENS after its effects have worn off.

According to Bennett et al., the validity of some reviews of TENS comes into question with relevance to the methodology used to assess pain treatment with physical modalities. Physical modalities may require a different evaluation to determine efficacy as any application of electrodes to the skin activates sensory fibers. These fibers are activated by light touch such as the application of an electrode to the skin, even one that is inactive as in placebo. "Light touch is known to affect Meissner's corpuscles and hair follicle endings (Somatosensory Study Guide Introduction)"[19].

The main consideration is that there is an urgent need to reach consensus as to how to address matters of low fidelity in reviews of the pain-relieving effects of physical modalities.

It should be noted that in the clinical application of the TENS treatment it invariably achieves its goal of relieving pain, improving mobility and enabling patients to continue with work and recreational activities providing patient's with control of their pain and their lives and thus improving their quality of life.

\section{Research Demonstrating Evidence for Physical Treat- ments}

In "An assessment of the efficacy of physical therapy and physical modalities for the control of chronic musculoskeletal pain" [20] an analysis of review articles and controlled clinical trials for temporomandibular disorders and similar chronic musculoskeletal pain disorders was carried out. "Although little evidence was found that any specific therapy had long-term efficacy greater than placebo, it was found that strong evidence exists that symptoms improve during treatment with most forms of physical therapy, including placebo". "When the frequency of significant between-group differences in trials that used placebo and waiting list control (i.e., no treatment) groups were compared, it was found that treatment was better than placebo in only $7 / 22$ trials, whereas treatment was almost always better than NO treatment" $[21,22]$. This difference was highly significant $(P=0.001)$ ". A similar analysis of trials that included more than one treatment group showed "that while equal amounts of treatment were usually associated with equal outcome" [14,15], "unequal treatment regimes led to unequal outcome" [21]. "The group that received the most therapy appeared to do best. In conclusion, it seems that patients are helped during the period that they are being treated with most forms of physical therapy. However, most of these therapies have not been shown to be more efficacious than placebo" [21].

\section{Research Demonstrating Evidence for Application of TENS Therapy}

In the defense of applying TENS treatments in many situations such as: pain in children, as a screening tool before spinal cord stimulation and in both acute and chronic pain conditions let us now examine the evidence for these applications and use of TENS.

Since the 1970s case histories of TENS use in children with reflex sympathetic dystrophies (now known as complex regional pain syndromes) were found to produce pain relief or even resolve the condition. "It is noted that researchers endorsed that TENS is a non-invasive physical modality that may provide excellent analgesia for some patients. It has been described in several case reports and series. None of these series describe TENS as universally effective, and there were no prospective, blinded trials of efficacy at that time. It was however generally accepted that in view of the modest cost, generally high acceptance by children, and remarkable safety of this device, it was almost always worthwhile giving a trial of TENS as part of a multidisciplinary approach to Complex Regional Pain Syndromes" [21,23].

In fact this conclusion was validated by a recent study by Mathew et al., in "TENS" for children's procedural pain [24]. "A three treatment group of patients in a double blind and placebo controlled trial was employed to investigate the effect of TENS treatment on pain produced by venipuncture. The three treatment groups consisted of TENS, placebo-TENS and control. Subjects were blocked into six 2-year age groups (ages: 5-17 years). During the period of the study, 896 children attending the outpatient laboratory of a general hospital were screened and 514 children completed the study. The data that was collected before venipuncture included expected pain and state anxiety. Following venipuncture, pain intensity was measured with a vertical Visual Analogue Scale (VAS) and pain affect was assessed with McGrath's faces scale. Significant main effects for treatment and age groups were obtained. Pain intensity and affect were lowest for the TENS group and highest for the control group. The pain scores were greatest for lower age groups and lowest for higher age groups". The 
results of this study support the use of TENS for children's pain and the need for interventions for children's procedural pain.

In a study "TENS trial may be used as screening tool prior to Spinal Cord Stimulator (SCS) implantation” by Leonard, Goffaux \& Marchand [25], there is evidence of a significant correlation between ability to tolerate TENS and SCS induced paraesthesias and between pre-SCS trial anxiety score and high pain score during SCS trial. The conclusion was that there is potential applicability of a TENS trial as a non invasive screening tool which may promote cost effectiveness and decrease unnecessary procedural risks to the patient by avoiding SCS trials, in select patients".

There has been some controversy regarding TENS and opioids in that it was purported that only low frequency TENS analgesic effects could be reversed by naloxone (opioid antagonist) but "a recent study on humans revealed that high frequency TENS can also be reversed by naloxone" This means that both low and high frequency TENS produce endogenous opioids/pain relief and therefore "individual patient choice may achieve the best results and this may also depend on the region where the TENS is applied" [26].

Animal studies on Sprague-Dawley rats have helped explain other mechanisms of TENS analgesia. "According to the researchers [26], TENS reduces pain through central mechanisms involving spinal cord and brainstem sites. Their studies "have demonstrated that TENS reduces chronic hyperalgesia induced by muscle inflammation. It was found that TENS reduced unilateral hyperalgesia when applied either ipsilaterally or contralaterally to the site of inflammation". "Chronic hyperalgesia due to muscle inflammation is a persistent feature of neuropathic pain and many human patients applying TENS for these conditions could and clinically do obtain pain relief" [27].

A paper dealing with investigations into the parameter selection (frequency, intensity and stimulation site) required to achieve maximal hypoalgesic effects using TENS was published in 2002 in Pain [28]. "Two hundred and forty participants were recruited in order to provide statistical analysis with $80 \%$ power at $\alpha=0.05$. Subjects were randomised to one of the six TENS groups, a control, and a sham TENS group ( $n=30,15$ males, 15 females, per group). TENS groups differed in their combinations of stimulation, frequency $(4 \mathrm{or} 110 \mathrm{~Hz})$, intensity ('to tolerance' or 'strong but comfortable') and stimulation site (segmental - over the distribution of the radial nerve or, extra segmental - over acupuncture point 'gall bladder 34', or a combination of both segmental and extra segmental). Pulse duration was fixed at $200 \mu$ s. Stimulation was delivered for $30 \mathrm{~min}$ and subjects were then monitored for a further $30 \mathrm{~min}$.

Mechanical Pain Threshold (MPT) was measured using a pressure algometer and taken from the first dorsal interosseous muscle of the dominant hand, ipsilateral to the stimulation site. MPT measures were taken, at baseline, and at $10 \mathrm{~min}$ intervals for $60 \mathrm{~min}$. Difference scores were analysed using repeated measures and one-way ANOVA and relevant post hoc tests.

The low frequency, high intensity, extra segmental stimulation produced a rapid onset hypoalgesic effect, which increased during the stimulation period and was sustained 30 mins post stimulation whereas high frequency, strong but comfortable intensity, segmental stimulation produced comparable hypoalgesic levels during stimulation which in this study was not sustained post stimulation".

Another published study "High frequency, high intensity TENS stimulation as treatment of pain after surgical abortion" [29] demonstrated that "TENS was comparable to intravenous conventional pharmacological treatment after surgical abortion. There was no difference between the groups (200 women randomized to TENS or conventional pharmacological treatment) with regard to pain relief according to the VAS pain score however the patients in the TENS group spent shorter time in the recovery ward (44 mins as opposed to 62 mins, $\mathrm{p}<0001)$. What is interesting about this study on TENS was that the TENS treatment was applied with a high intensity (20-60 ma) for one minute and repeated once if insufficient pain relief occurred. This study highlights the differences that intensity, frequency, duration and site may achieve in pain relief hence making it difficult to compare studies on TENS. Hence TENS is a versatile treatment for many different types of pain, acute and chronic, in different patients".

Tolerance to TENS also occurs leaving no doubt that application of TENS produce opioids. This was validated in animal models that demonstrate that repeated TENS application produces tolerance and cross-tolerance at spinal opioid receptors. The aim of the study "An investigation of the development of analgesic tolerance to TENS in humans" was to examine whether repeated application of TENS produces analgesic tolerance in humans [30]. "One hundred healthy subjects were randomly assigned to 1 of 4 groups: control, placebo, low-frequency $(4 \mathrm{~Hz})$ or high-frequency $(100 \mathrm{~Hz})$ TENS. TENS was applied daily for 5 days to the non-dominant upper limb; Pressure-Pain Threshold (PPT) measurements were recorded before and after TENS. Temporal summation to mechanical stimulation (increasing strength of signals in each neuronal fibre) was recorded on days 1 and 5, before and after TENS. Diffuse Noxious Inhibitory Control (DNIC) was tested on day 5 using the cold pressor test and PPT measurements. There was an initial increase in PPTs in both low- and high-frequency TENS groups when compared with placebo or control groups. However, by day 5 this TENS-induced increase in PPT did not occur, and there was no difference between active TENS and placebo or control groups. High-frequency TENS decreased temporal summation on day 1 when compared with day 5 . DNIC increased the PPT similarly in all groups. These data suggest that repeated daily application of TENS results in a decrease in its hypoalgesic effect by the fifth day and that the tolerance-like effect to repeated TENS results from tolerance at centrally located opioid receptors. The lack of change in DNIC response suggests that TENS and DNIC utilize separate pathways to produce analgesia". "Tolerance can however be reduced by alternating high and low frequency TENS” according to Chandra \& Sluka [30].

In "Predicting outcome of TENS in chronic pain: A prospective, randomized, placebo controlled trial" by Oosterhof et al., [31], an attempt was made to explore the factors that would predict results of TENS treatment in chronic pain.

"A randomized, placebo-controlled trial $(n=163)$, comparing high frequency TENS $(n=81)$ with sham TENS $(n=82)$ and patients' satisfaction (willingness to continue treatment: yes or no) and pain intensity (VAS) were used as outcome measures. The origin of pain and cognitive coping strategies were evaluated as possible predictors for result of TENS treatment.

\section{Results}

Fifty-eight percent of the patients in the TENS group and $42.7 \%$ of the sham-TENS group were satisfied with treatment result (chi square $=3.8, \mathrm{p}=0.05$ ). No differences were found for pain intensity. Patients diagnosed with osteoarthritis and related disorders (especially of the vertebral column) or peripheral neuropathic pain were less satisfied with high frequency TENS). Injury of bone and soft tissue (especially postsurgical pain disorder) provided the best results. It 
was concluded, that predicting the effect of high frequency TENS in chronic pain depends on the choice of outcome measure. Predicting patients' satisfaction with treatment result is related to the origin of pain. Predicting pain intensity reflects mechanisms of pain behaviour and perceived control of pain, independent of treatment modality".

"At its most basic level, satisfaction is a comprehensive evaluation of several dimensions of health care based on patient expectations and provider and treatment performance" [32]. "From the patient's perspective, satisfaction includes many facets such as accessibility, convenience, availability of resources, continuity of care, efficacy, finances, humaneness, information gathering, information giving, pleasantness of surroundings, quality and competence" [33].

A Patient Treatment Satisfaction Questionnaire (PTSS) was developed by Evans, Trudeau, Mertzanis et al., [34] that validates the importance of analyzing patient satisfaction with their treatment in acute and chronic pain.

"As an outcomes measure, patient satisfaction allows health care providers to assess the appropriateness of treatment according to patient expectations. In chronic diseases, where patients must live with treatment, patient satisfaction may be the distinguishing outcome among treatments with comparable efficacy" [35]. "Evidence also suggests that patient satisfaction may be more sensitive to change than quality of life in clinical trials in chronic diseases" [36].

As several types of TENS, based on different combinations of frequency, pulse duration and intensity exist, a study was executed to evaluate the precise mechanism of action and the relevance of combinations of stimulus parameters.

In "Pain reducing effect of three types of transcutaneous electrical nerve stimulation in patients with chronic pain: a randomized crossover trial" [8].

"In the randomized, single blinded crossover trial patients' received two times a 2-week period of daily TENS treatment, separated by a washout period of 2 weeks. In total, 180 chronic pain patients were randomized into three groups. In group 1, high frequency, low intensity TENS (HFT) was compared with high frequency, High Intensity TENS (HIT). In groups 2 and 3, HFT and HIT were compared with a Control TENS (COT). The order of applying the different modalities of TENS in each group was also randomized.

Primary outcome was the patient's overall assessment of effectiveness and pain reduction (VAS). No differences were found in patient's assessment or pain reducing effect between the three groups, indicating no superiority of one type of TENS. In total, $56 \%$ continued TENS after the 2-week treatment period. At 6 months, $42 \%$ of all patients still used TENS.

It was thus concluded that there were no differences in effectiveness for the three types of TENS used in this study. Because no placebo group was included, no definite conclusions on effectiveness of TENS in general in the treatment of chronic pain could be made".

However the value of this study indicates that effectiveness of TENS is achieved by different parameters and may be used in different conditions in different patients. Also almost half the patients in these study groups continued to use the TENS indicating patient satisfaction with TENS pain relief.

Another study examined the relationships between patients, stimulator and out-come variables in a large number of chronic pain patients utilizing TENS on a long-term basis. This has particular relevance for medical insurance companies - as many patients request these companies to assist in the purchasing of 'own devices' for patients to use when necessary for pain relief. The outcome of "An in-depth study of long-term users of transcutaneous electrical nerve stimulation revealed that in 107 patients, $47 \%$ of patients found TENS reduced their pain by more than half, TENS analgesia was rapid both in onset (less than 0.5 hours in $75 \%$ patients) and in offset (less than 0.5 hours in $51 \%$ patients), one-third of patients utilised TENS for over 61 hours/week; pulse frequencies between 1 and $70 \mathrm{~Hz}$ were utilised by $75 \%$ of patients; $44 \%$ of patients benefitted from burst mode stimulation [37]. The fact that patients continue to use the device on their own when and wherever they require it, is a strong indication for supporting patients who feel TENS improves their quality of life and has the added value that it does not produce side effects".

"A randomized comparative trial of acupuncture versus transcutaneous electrical nerve stimulation for chronic back pain in the elderly was performed on sixty patients aged 60 or over with back pain for at least 6 months that were recruited from General Practitioner referrals and randomized to 4 weeks of treatment with acupuncture or Transcutaneous Electrical Nerve Stimulation (TENS) [38]. All the above treatments were administered by the same physiotherapist and both groups had the same contact with him. The following were measured at baseline, completion and at a 3-month follow-up by an independent observer blinded to treatment received: (1) pain severity on Visual Analogue Scale (VAS), (2) pain subscale of Nottingham Health Profile (NHP), (3) number of analgesic tablets consumed in previous week, (4) spinal flexion from C7 to S1. Thirty-two patients were randomized to acupuncture and 28 to TENS; only three withdrew (two from acupuncture, one from TENS). Significant improvements were shown on VAS $(\mathrm{P}<0.001)$, NHP $(\mathrm{P}<0.001)$ and tablet count $(\mathrm{P}<0.05)$ between baseline and completion in both groups, these improvements remaining significant comparing baseline with follow-up with a further non-significant improvement in VAS and NHP in the acupuncture group. The acupuncture but not the TENS patients showed a small but statistically significant improvement $(\mathrm{P}<0.05)$ in mean spinal flexion between baseline and completion which was not maintained at follow-up. Thus in these elderly patients with chronic back pain both acupuncture and TENS had demonstrable benefits that outlasted the treatment period. Acupuncture may improve spinal flexion".

There is also a possibility of using TENS for cancer bone pain and a feasibility study was embarked on by Bennett, Johnson, Brown et al., [39] with patients recruited from palliative care services. "Cancer bone pain is common and severe and may also involve neural hyper excitability. In animal studies there is a suggestion that TENS can reduce hyperalgesia. The results in the cancer bone pain study indicated that TENS has the potential to decrease pain on movement more than pain on rest".

It is suggested that if patients achieve pain relief in any condition including chronic pain secondary to cancer, TENS is worth the consideration and it should be recommended as one of a combination of therapies in treatment. It is a simple task to provide this type of patient the opportunity to test TENS in their condition and if there is no improvement or indeed irritation then it is easily discontinued and there are usually nil side effects.

Previous studies and meta-analysis of the efficacy of Electrical Nerve Stimulation (ENS) for the treatment of chronic pain of multiple etiologies have produced mixed results. A recent study by Johnson M. Martinson [40] "endeavoured to determine whether ENS is an effective treatment for chronic musculoskeletal pain by using statistical 
techniques that permit accumulation of a sample size with adequate power. Randomized, controlled trials published between January 1976 and November 2006 were obtained from the National Libraries of Medicine, EMBASE and the Cochrane Library. Prospective, placebo-controlled studies using any modality of ENS to treat chronic musculoskeletal pain in any anatomical location were included. The main outcome measure was pain at rest. The use of statistical methods to enhance data extraction and a random-effects meta-analysis to accommodate heterogeneity of ENS therapies permitted an adequate number of well designed trials of ENS to be included in the meta-analysis. A total of 38 studies in 29 papers, which included 335 placebo, 474 ENS and 418 crossover (both placebo and at least one ENS treatment) patients, met the selection criteria. The overall results showed a significant decrease in pain with ENS therapy using a random-effects model $(\mathrm{p}<0.0005)$ ". These results indicate that ENS is an effective treatment modality for chronic musculoskeletal pain and that previous, equivocal results may have been due to underpowered studies.

Patients are individuals with specific preferences when using electrical currents for pain relief. In the study - "The consistency of pulse frequencies and pulse patterns of Transcutaneous Electrical Nerve Stimulation (TENS) used by chronic pain patients, the results record the consistency of Transcutaneous Electrical Nerve Stimulation (TENS) in pulse frequency and pulse pattern used by 13 chronic patients over a 1 year period. The results show that patients prefer specific pulse frequencies and pulse patterns unique to the individual and that they return to such frequencies and patterns on subsequent treatment sessions. Pulse frequencies and pulse patterns were not related to the cause and site of pain, a finding consistent with a previous study in this laboratory. This observation, coupled with the large variability in pulse frequencies and pulse patterns used between individuals, implies that patients prefer such frequencies and patterns for reasons of comfort which may not be related to mechanisms specific to the pain system" [32].

\section{Recommendations for the Use of TENS}

As a result of the evaluations from previous studies and the clinical evidence that presents itself daily in physiotherapy practice and on the information acquired from patients, recommendations are suggested for the use and promotion of TENS. Patient satisfaction scores in TENS treatment may be more significant than visual analogue scale scores.

It is therefore proposed that patients should first consult their physiotherapist before embarking on a TENS trial, decided upon by the physiotherapist for a period of not less than three days. This may determine if these patients are responders to TENS. The therapist or medical advisory would advise and determine the best application of this device e.g., where to place the electrodes for their particular problem, parameters of application and duration of usage.

Optimal pain relief occurs during treatment enabling tolerance of treatment and exercise programmes. This enables patients to understand that, with self application of TENS they could participate in both recreational and occupational activities whenever it is suitable, with less pain.

It has been noted that prolonged pain relief may also be obtained post TENS treatment in certain patients in many situations. Elderly patients with chronic back pain benefit from both acupuncture and TENS that outlasts the treatment period. The fact that patients continue to use the device on their own when and wherever they require it, is a strong indication for supporting patients who feel TENS improves their quality of life and does not produce side effects. Tolerance to TENS may also occur and this can be avoided by alternating the frequencies.

TENS use in children with reflex sympathetic dystrophies (Complex Regional Pain Syndromes - CRPS) was found to produce pain relief or even resolve the condition in some patients. Children that exhibit early signs of CRPS may respond rapidly to TENS, even within three treatments (clinical observation). TENS may also be used for procedural pain in children and by inference in adults as well as studies that have demonstrated the use and value of TENS in post surgical abortion, to name one among many other post surgical situations.

TENS may also be used as a screening tool prior to spinal cord stimulation implantation. Electrical Nerve Stimulation is an effective treatment modality for some patients with chronic musculoskeletal pain. Patients should be encouraged to use electrical stimulation if they are responding with improved pain relief and mobilisation. Electrical treatments are known to improve circulation as well as pain and it may enable the medical advisor to reduce medication in these circumstances.

There is large variability in pulse frequencies and pulse patterns used between individuals, implying that patients prefer such frequencies and patterns for reasons of comfort that may not be related to mechanisms specific to the pain system. Patients should be encouraged to use the frequency that provides them with "best treatment".

TENS treatment may be affected by type and site of application, optimal treatment duration and intensities - all of these being an individual application depending on the condition, symptoms, area of treatment and the patient. TENS use is most effective when applied for at least 40 mins. Both high frequency and low frequency TENS produces endorphins positively influencing pain relief.

\section{Which Patients Should Not Use a TENS Device?}

TENS is usually a device that can be used by many patients in most conditions however there are patients with nerve disturbances from neuropathic pain or sensitisation of either or both the peripheral or central nervous system that may discover that TENS could irritate the pain or make the condition worse. "Neuropathic pain is caused by a lesion or dysfunction of the peripheral or central nervous system" [41]. The reason for TENS producing this effect is due to mechanical stimulation of the larger A beta fibres that have now become sensitised due to prolonged pain and neuropathic discharge from some or all of these sensory fibres either in the region of injury or centrally in the spinal cord. When these fibres become sensitised vibration, massage, movement or A beta fibre stimulation increases dysaesthesias, pain and allodynia and may produce or irritate neuropathic pain. A patient who has this condition will notice an immediate discomfort or increased pain and the treatment should then be discontinued immediately (clinical observation).

Patients who are pregnant should not use TENS over the pregnant uterus but TENS has been used in spinal or peripheral regions for relief of musculoskeletal conditions during pregnancy without any deleterious effects to the patient or foetus. In fact TENS is used and encouraged during labour to relieve back and low abdominal pains.

Patients who have pacemakers or peripheral and or central nerve stimulators should be aware that the TENS device may disturb electrical or magnetic fields and should consult their attending physician before considering applying TENS in these circumstances. 
Citation: Berger P (2017) Validation of the Use of Transcutaneous Electrical Nerve Stimulation for Patients with Pain and Recommendations for its Use by Physiotherapists. J Anesth Clin Care 4: 019.

\section{Side Effects of the Use of a TENS Device}

Side effects from TENS use are uncommon. Mostly, if any side effects do occur, it is the skin that may be sensitive to the electrodes. Prior to placement of electrodes (pads with gel), the skin should be thoroughly cleaned and dried and then cleaned and dried once again after use. Some patients may become allergic or sensitive to these gel pads but most patients are able to find the electrodes that best suit their particular skin type. If an allergy arises, then anti-histamine type creams can be applied locally to relieve the condition. Electrode placement can readily be changed if skin irritation does occur without compromising the treatment plan.

Sometimes over use of the TENS may create muscle soreness if the muscles have been activated over a prolonged period due to twitching in the muscles under the electrodes. This muscle soreness is temporary and does not cause any lasting ill effects.

\section{Conclusion}

TENS is a safe, effective, economical and simple device to use for the relief of pain with minimal side effects. It may enable the patient to reduce their medication (only on medical advice) which will also limit unwanted side effects. TENS can be used in combination with other therapies both physical and pharmalogical without interfering with other treatment. Most treatment is applied in combination that will address all aspects of the patient's condition and TENS has been found to be a beneficial adjunctive treatment that assists hypoalgesia.

\section{References}

1. Sluka KA (2009) Transcutaneous Electrical Nerve stimulation and Interferential Therapy. In: Craik RL (ed.). Mechanisms and Management of Pain for the Physical Therapist, International Association for the Study of Pain, Washington, DC, USA.

2. Melzack R (1999) From the gate to neuromatrix. Pain 6: 121-126.

3. Melzack R, Wall PD (1965) Pain mechanisms: a new theory. Science 150: 971-979.

4. Bennett MI, Hughes N, Johnson MI. Methodological quality in randomised controlled trials of transcutaneous electric nerve stimulation for pain: Low fidelity may explain negative findings. Pain 152: 1226-1232.

5. Moore SR, Shurman J (1997) Combined neuromuscular electrical stimulation and transcutaneous electrical nerve stimulation for treatment of chronic back pain: a double-blind, repeated measures comparison. Arch Phys Med Rehabil 78: 55-60.

6. Boutron I, Moher D, Altman DG, Schulz KF, Ravaud P, et al. (2008) Extending the CONSORT statement to randomized trials of nonpharmacologic treatment: explanation and elaboration. Ann Intern Med 148: 295-309.

7. Al-Smadi J, Warke K, Wilson I, Cramp AFL, Noble G, et al. (2003) A pilot investigation of the hypoalgesic effects of transcutaneous electrical nerve stimulation upon low back pain in people with multiple sclerosis. Clin Rehabil 17: 742-749.

8. Köke AJ, Schouten JS, Lamerichs-Geelen MJ, Lipsch JS, Waltje EM, et al. (2004) Pain reducing effect of three types of transcutaneous electrical nerve stimulation in patients with chronic pain: a randomized crossover trial. Pain 108: $36-42$.

9. Lewis B, Lewis D, Cumming G (1994) The comparative analgesic efficacy of transcutaneous electrical nerve stimulation and a non-steroidal anti-inflammatory drug for painful osteoarthritis. Br J Rheumatol 33: 455-460.

10. Lewis D, Lewis B, Sturrock RD (1984) Transcutaneous electrical nerve stimulation in osteoarthrosis: a therapeutic alternative?. Ann Rheum Dis 43: 47-49.

11. Jensen H, Zesler R, Christensen T (1991) Transcutaneous electrical nerve stimulation (TNS) for painful osteoarthrosis of the knee. Int J Rehabil Res 14: $356-358$.
12. Cheing GL, Tsui AY, Lo SK, Hui-Chan CW (2003) Optimal stimulation duration of tens in the management of osteoarthritic knee pain. J Rehabil Med 35: 62-68.

13. Milne S, Welch V, Brosseau L, Saginur M, Shea B, et al. (2001) Transcutaneous electrical nerve stimulation (TENS) for chronic low back pain. Cochrane Database Syst Rev.

14. Claydon LS, Chesterton LS, Barlas P, Sim J (2011) Dose-specific effects of transcutaneous electrical nerve stimulation (TENS) on experimental pain: $A$ systematic Review. Clin J Pain 27: 635-647.

15. Hsueh TC, Cheng PT, Kuan TS, Hong CZ (1999). The immediate effectiveness of electrical nerve stimulation and electrical muscle stimulation on myofascial trigger points. Am J Phys Med Rehabil 76: 471-476.

16. Abelson K, Langley GB, Sheppeard H, Vlieg M, Wigley RD (1983) Transcutaneous electrical nerve stimulation in rheumatoid arthritis. N Z Med J 96: 156-158.

17. Grimmer K (1992) A controlled double blind study comparing the effects of strong burst mode TENS and high rate TENS on painful osteoarthritic knees. Aust J Physiother 38: 49-56.

18. Mannheimer C, Carlsson CA (1979) The analgesic effect of transcutaneous electrical nerve stimulation (TNS) in patients with rheumatoid arthritis. A comparative study of different pulse patterns. Pain 6: 329-334.

19. http://healthcaresciencesocw.wayne.edu/sensory/1_5.htm

20. Feine JS, Lund JP (1997) An assessment of the efficacy of physical therapy and physical modalities for the control of chronic musculoskeletal pain. Pain 71: 5-23.

21. Ashwal S, Tomasi L, Neumann M, Schneider S (1988) Reflex sympathetic dystrophy syndrome in children. Pediatr Neurol 4: 38-42.

22. Wilder RT, Berde CB, Wolohan M, Vieyra MA, Masek BJ, et al. (1992) Reflex sympathetic dystrophy in children. Clinical characteristics and follow-up of seventy patients. J Bone Joint Surg Am 74: 910-919.

23. Lander J, Fowler-Kerry S (1993) TENS for children's procedural pain. Pain 52: $209-216$.

24. Mathew L, Winfree C, Miller-Saultz D, Sonty N (2010) Transcutaneous electrical nerve stimulator trial may be used as a screening tool prior to spinal cord stimulator implantation. Pain 150: 327-331.

25. Leonard G, Goffaux P, Marchand S (2010) Deciphering the role of endogenous opioids in high-frequency TENS using low and high doses of naloxone. Pain 151: 215-219.

26. Ainsworth L, Budelier K, Clinesmith M, Fiedler A, Landstrom R, et al. (2006) Transcutaneous electrical nerve stimulation (TENS) reduces chronic hyperalgesia induced by muscle inflammation. Pain 120: 182-187.

27. Chesterton LS, Barlas P, Foster NE, Lundeberg T, Wright CC, et al. (2002) Sensory stimulation (TENS): effects of parameter manipulation on mechanical pain thresholds in healthy human subjects. Pain 99: 253-262.

28. Liebano RE, Rakel B, Vance CG, Walsh DM, Sluka KA (2011) An Investigation of the Development of Analgesic Tolerance to Transcutaneous Electrical Nerve Stimulation (TENS) in Humans. Pain 152: 335-342.

29. Platon B, Andréll P, Raner C, Rudolph M, Dvoretsky A, et al. (2010) High-frequency, high-intensity transcutaneous electrical nerve stimulation as treatment of pain after surgical abortion. Pain 148: 114-119.

30. Chandran P, Sluka KA (2003) Development of opioid tolerance with repeated transcutaneous electrical nerve stimulation administration. Pain 102: 195201.

31. Oosterhof J, Samwel HJ, de Boo TM, Wilder-Smith OH, Oostendorp RA, et al. (2008) Predicting outcome of TENS in chronic pain: a prospective, randomized, placebo controlled trial. Pain 136: 11-20.

32. Johnson MI, Ashton CH, Thompson JW (1991) An in-depth study of long-term users of transcutaneous electrical nerve stimulation (TENS). Implications for clinical use of TENS. Pain 44: 221-229. 
Citation: Berger P (2017) Validation of the Use of Transcutaneous Electrical Nerve Stimulation for Patients with Pain and Recommendations for its Use by Physiotherapists. J Anesth Clin Care 4: 019.

33. Steiber SR, Krowinski WJ (1990) Measuring and managing patient satisfaction. American Hospital Publishing, Chicago IL, USA.

34. Evans CJ, Trudeau E, Mertzanis P, Marquis P, Peña BM, et al. (2004) Development and validation of the pain treatment satisfaction scale (ptss): a patient satisfaction questionnaire for use in patients with chronic or acute pain. Pain 112: 254-266.

35. Weaver MJ, Ow CL, Walker DJ, Degenhardt EF (1993) A questionnaire fo patients' evaluations of their physicians' humanistic behaviors. J Gen Intern Med 8: 135-139.

36. Weinberger M1, Oddone EZ, Henderson WG (1996) Does increased access to primary care reduce hospital readmissions? Veterans Affairs Cooperative Study Group on Primary Care and Hospital Readmission. N Engl J Med 334: 1441-1447.

37. Johnson MI, Ashton CH, Thompson JW (1991) The consistency of pulse frequencies and pulse patterns of Transcutaneous Electrical Nerve Stimulation (TENS) used by chronic pain patients. Pain 44: 231-234.
38. Grant SJ, Bishop-Miller J, Winchester DM, Anderson M, Faulkner S (1999) A randomized comparative trial of acupuncture versus transcutaneous electrical nerve stimulation for chronic back pain in the elderly. Pain 82: 9-13.

39. Bennett MI, Johnson MI, Brown SR, Radford H, Brown JM, et al. (2010) Feasibility study of Transcutaneous Electrical Nerve Stimulation (TENS) for cancer bone pain. J Pain 11: 351-359.

40. Johnson M, Martinson M (2007) Efficacy of electrical nerve stimulation for chronic musculoskeletal pain: a meta-analysis of randomized controlled trials. Pain 130: 157-165.

41. Merskey H, Bogduk N (1994) Classification of Chronic Pain. Seattle: IASP Press, Seattle, WA, USA 